\title{
La compétition et la division ne font pas avancer la recherche
}

\section{(Q) OpenEdition}

Journals

Édition électronique

URL : http://journals.openedition.org/developpementdurable/17044

DOI : 10.4000/developpementdurable.17044

ISSN : 1772-9971

Éditeur

Association DD\&T

Référence électronique

"La compétition et la division ne font pas avancer la recherche ", Développement durable et territoires [En ligne], Vol. 11, $\mathrm{n}^{\circ} 1$ | Avril 2020, mis en ligne le 30 avril 2020, consulté le 24 septembre 2020. URL : http://journals.openedition.org/developpementdurable/17044; DOI : https://doi.org/10.4000/ developpementdurable.17044

Ce document a été généré automatiquement le 24 septembre 2020

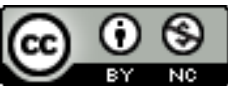

Développement Durable et Territoires est mis à disposition selon les termes de la licence Creative Commons Attribution - Pas d'Utilisation Commerciale 4.0 International. 


\section{La compétition et la division ne font pas avancer la recherche}

Cet éditorial a été rédigé par le comité de rédaction dans son ensemble.

1 Comme de nombreux services publics, et notamment l'hôpital public, la recherche et l'enseignement supérieur public sont sous tension. À l'instar de la nature, de la biodiversité ou du climat, le terme d'effondrement paraît aujourd'hui le plus adéquat pour décrire la situation. Dans tous les cas, les origines anthropiques de ces multiples crises révèlent la capacité de l'humanité à détruire le vivant (ainsi que son habitat) tout comme ce qu'il a construit. Ici, comme ailleurs, c'est la même destruction qui est à l'œuvre.

2 À l'heure d'une nouvelle décennie qui s'ouvre par la prise de conscience de l'urgence à repenser radicalement nos rapports à la planète, les professionnels de la recherche et de l'enseignement supérieur, en première ligne des combats environnementaux et sanitaires, travaillent désormais dans l'incertitude radicale, épuisés par vingt ans de réformes néolibérales, répondant aux injonctions de la mise en concurrence, des classements internationaux aux critères parfois aberrants et/ou incohérents, des laboratoires et formations dont l'«excellence » n'est attestée que par ces mêmes critères, etc.

3 La recherche tente aujourd'hui de survivre face aux règles du new public management qui se traduisent par un financement largement fondé sur la rédaction de projets, des évaluations permanentes et des bilans, dont le caractère chronophage n'a d'égal que celui lié à leur répétition, inlassablement. Aspiré par son écran d'ordinateur, le chercheur sonde dans quelle niche il pourra désormais s'inscrire pour que sa recherche apparaisse comme innovante aux yeux des instances évaluatrices, étudie de quelle manière insuffler un esprit de rentabilité dans ses activités, aussi éloignées soient-elles des possibilités de concrétisation marchande, en justifiant la pertinence de cette recherche par une masse de livrables, réduisant jour après jour le temps consacré à des recherches singulières mais d'utilité publique, à préparer ses cours pour des étudiants préoccupés, notamment par la précarité et leur avenir. 
4 De la même manière que dans les hôpitaux publics, la notion de service public tend à s'effacer au profit de normes managériales du secteur privé. Les universités, les écoles et les organismes de recherche sont aujourd'hui les plus grands employeurs de contractuels parmi les institutions étatiques. L'enseignement supérieur et la recherche tendent à être soumis aux lois de la performance, de la rigueur budgétaire, de l'efficience, du manque de personnels, des turnovers, du surmenage, des désaffections, au risque de la perte de sens. La souffrance au travail est désormais une réalité quotidienne pour nombre de personnels de l'ESR.

$5 \quad$ Et l'avenir de nos doctorants? Aussi sombre que celui que l'on prédit à nos oiseaux, aux pollinisateurs et flore adventice? L'âge moyen de recrutement des maîtres de conférences ou des chargés de recherches au CNRS est aujourd'hui de 36 ans (32 ans en 2004), et encore, faut-il être recruté! Au regard du très faible nombre de postes mis au concours chaque année, ce sont des centaines de chercheurs qui s'ancrent dans une précarité mortifère. Sélection naturelle?

6 Et l'égalité/équité dans tout ça? Le monde académique ne cesse d'afficher sa forte préoccupation en matière d'égalité femmes-hommes, avec l'obligation de représentation équilibrée dans les différentes instances, la multiplication des campagnes menées contre les violences sexistes et le harcèlement. Mais de nombreuses chercheuses dénoncent ces mesures de simple affichage pour les classements internationaux qui s'appuient sur une approche individuelle, plutôt que structurelle, des inégalités.

7 Dans ce contexte, pour " rétablir l'attractivité des carrières scientifiques », comme le dit la ministre Frédérique Vidal, le gouvernement projette la Loi de Programmation Pluriannuelle de la Recherche (LPPR). La meilleure description de cette loi est encore celle qu'en faisait Antoine Petit, le PDG du CNRS dans le quotidien Les Échos, le 26 Novembre 2019: «Il faut une loi ambitieuse, inégalitaire - oui, inégalitaire, une loi vertueuse et darwinienne, qui encourage les scientifiques, équipes, laboratoires, établissements les plus performants à l'échelle internationale $»^{1}$. Sans que rien ne soit encore arrêté définitivement, les pistes de réflexion envisagent par exemple : de tenir compte de la " performance » des collègues dans la revalorisation de leurs salaires (et le financement de leur recherche), de remettre en question la référence à un volume horaire statutaire identique pour tous les enseignants-chercheurs, ou encore la mise en place d'une tenure-track, procédure anglo-saxonne qui vise le "recrutement conditionnel ou titularisation conditionnelle ». D'une durée de 5 à 7 ans, cette période probatoire se terminerait non pas par une titularisation systématique, mais par une procédure d'évaluation suivant des "critères d'excellence internationaux ", tenant compte aussi bien des livrables publiés que de la capacité à obtenir des financements lors des appels à projet.

8 Cette réforme poursuit méthodiquement la mise en compétition de tous contre tous et l'édification de la rentabilité (quantifiée, voire monétaire) comme critère d'organisation de l'Enseignement Supérieur et de la Recherche. La liberté académique se trouve remise en question par des impulsions autoritaires sur les collègues de l'enseignement et de la recherche, et la multiplication de statuts instables qui favorise la flexibilité/adaptabilité et partant, le contrôle du secteur. C'est donc dans ce double cadre de lecture qu'il faut considérer la lutte contre la LPPR : le néolibéralisme poursuit sa colonisation des secteurs de l'État, notamment celui de l'enseignement et de la recherche, autant qu'il poursuit sa colonisation de la nature. 


\section{NOTES}

1. Cette déclaration a suscité l'ire des chercheurs qui récoltèrent près de 15000 signatures dans une pétition-réponse intitulée « Lettre à MM. Petit et Macron, et à tous les tenants de la compétition comme mode de fonctionnement des sociétés humaines ». https://www.petitions.fr/recherche__non_a_une_loi_inegalitaire. 